\title{
Contribution à la taxonomie et à l'écologie des chênes du Berry
}

\author{
G. GRANDJEAN, P. SIGAUD \\ E.N.I.T.E.F., Domaine des Barres, F 45290 Nogent-sur-Vernisson
}

\begin{abstract}
Résumé
Des observations morphologiques fines de rameaux, feuilles et fructifications sont effectuées sur 419 chênes, dans des populations sympatriques ou allopatriques. Dix-neuf caractères, divisés en un total de 82 classes, sont ainsi recensés. L'analyse factorielle des correspondances met en évidence 3 pôles très nets reliés par quelques intermédiaires. Ces 3 pôles correspondent à Quercus robur L., Quercus petraea (Matt.) Liebl., et Quercus pubescens Willd. La rareté des individus réellement intermédiaires permet de rattacher la plupart des arbres à l'un des trois taxons.

Les variations des longueurs des pédoncules et des pétioles sont étudiées séparément dans l'arbre et le peuplement.

Parallèlement, des stations sont isolées par une étude phytoécologique, et sont corrélées à l'étude taxonomique. Il ressort de cette confrontation que :

- la population de chênes est une indicatrice du milieu,

- spéciation taxonomique et séparation écologique vont de pair.

Quoique les échanges interspécifiques semblent limités à l'heure actuelle, l'hypothèse de l'introgression apparaît malgré tout plausible.
\end{abstract}

\section{Introduction}

Les chênes ont toujours occupé une place privilégiée en Europe, tant à cause de leurs qualités technologiques que de la valeur quasi-sentimentale qui leur est attribuée.

Ils restent malgré cela très méconnus des forestiers et des chercheurs, et des questions sont régulièrement posées à leur sujet.

La première et principale est d'ordre botanique : si l'existence des 3 grands chênes européens : Quercus robur L., Quercus petraea (Matt.) Liebl. et Quercus pubescens Willd, ne fait plus guère de doute, il n'en est pas de même des individus supposés intermédiaires; les innombrables formes qui ne correspondent pas aux descriptions classiques ont été interprétées tantôt comme des sous-espèces, variétés ou formes, tantôt comme des hybrides.

Plusieurs travaux ont été réalisés ces dernières décennies à l'étranger, et une théorie unificatrice a été proposée, celle de l'hybridation introgressive. Selon celle-ci, après interfécondation, les croisements suivants seraient surtout des back-cross, chaque 
espèce «phagocytant » ainsi peu à peu une partie du génôme d'une ou des deux autres espèces. Les individus hybrides formés auraient des caractères intermédiaires ${ }^{(1)}$ entre ceux des espèces parentales, et occuperaient des localisations écologiques différentes des exigences des parents.

Il s'agissait donc de savoir ce qu'il en est dans le Berry où le chêne constitue un matériel forestier important n'ayant jamais, à notre connaissance, fait l'objet d'études fines.

On envisagera successivement dans cet article les aspects botanique (taxonomie et variation), écologique et phytogénétique.

\section{Taxonomie}

\subsection{Matériel et méthodes d'étude}

\subsection{Méthodologie générale}

Le but du travail est de vérifier la validité de la classification des chênes. La mise en évidence d'éventuels intermédiaires nécessite une étude morphologique fine des arbres, et non une simple reconnaissance pratique.

Cette taxonomie se fait en 3 étapes: description des U.T.O. (Unités Taxonomiques Opérationnelles, ici les arbres), estimation des ressemblances, et classement en groupes.

Il faut donc au départ un nombre important de caractères différentiels indépendants, ou supposés tels, dont le regroupement a posteriori permettra de définir les taxons. Les caractères en question concernent le rameau de l'année, la feuille, et la fructification.

\subsection{Région d'étude}

L'étude a été réalisée en Berry (départements du Cher et de l'Indre), au Centre de l'Office National des Forêts de Bourges.

Le climat est de type océanique dégradé, avec une tendance continentale plus ou moins prononceee.

Le domaine floristique est atlantique, secteur ligérien (FlaHAUT).

Les formations géologiques appartiennent aux terrains sédimentaires du Bassin Parisien. Un vaste plateau central de calcaires jurassiques, la Champagne berrichonne, est limité au Nord-Ouest par la cuvette tertiaire, acide et humide de Sologne, et à l'Est par les collines du Sancerrois.

Les sols sont très variés, depuis les rendzines de la vallée du Cher jusqu'aux sols podzoliques solognots, en passant par toutes les intensités d'hydromorphie.

(1) Dans cet article, lc terme « intermédiaire » ne sigrifie pas : à ćgalc distance. Le génome de l'une des espèces prédomine en général dans les produits de l'introgression. 
Il s'agit donc d'une région petite mais diversifiée, où les trois chênes : sessile, pédonculé, pubescent, coexistent.

\subsection{Choix des placettes}

L'implantation des placettes d'échantillonnage des arbres obéit à certains impératifs d'ordre sylvicole et de gestion pour déterminer un choix de l'essence objectif (en l'occurrence chêne pédonculé ou sessile) dans les taillis-sous-futaie en conversion. Les placettes ont donc été installées en forêts soumises, traitées en taillis-sous-futaie, et si possible après une coupe de taillis. Les arbres sont des réserves, modernes et anciens.

Elles sont établies d'après l'écologie et non le peuplement, de manière à représenter les grands milieux berrichons. Leur surface n'est pas délimitée mais impérativement homogène sur le plan stationnel, et l'effectif est variable (de 5 à 40 arbres).

$\mathrm{Au}$ total, 32 placettes rassemblent 419 arbres.

L'échantillon élémentaire est l'arbre. Les arbres sont numérotés sur le terrain. Sur chacun, 5 rameaux, 10 feuilles, 20 pédoncules sont tirés au fusil ou cueillis à l'échenilloir en périphérie du houppier de lumière (les feuilles ont ainsi des caractères bien marqués sans pour autant être abrasé par le vent). Ces spécimens sont dépouillés au bureau $^{(2)}$.

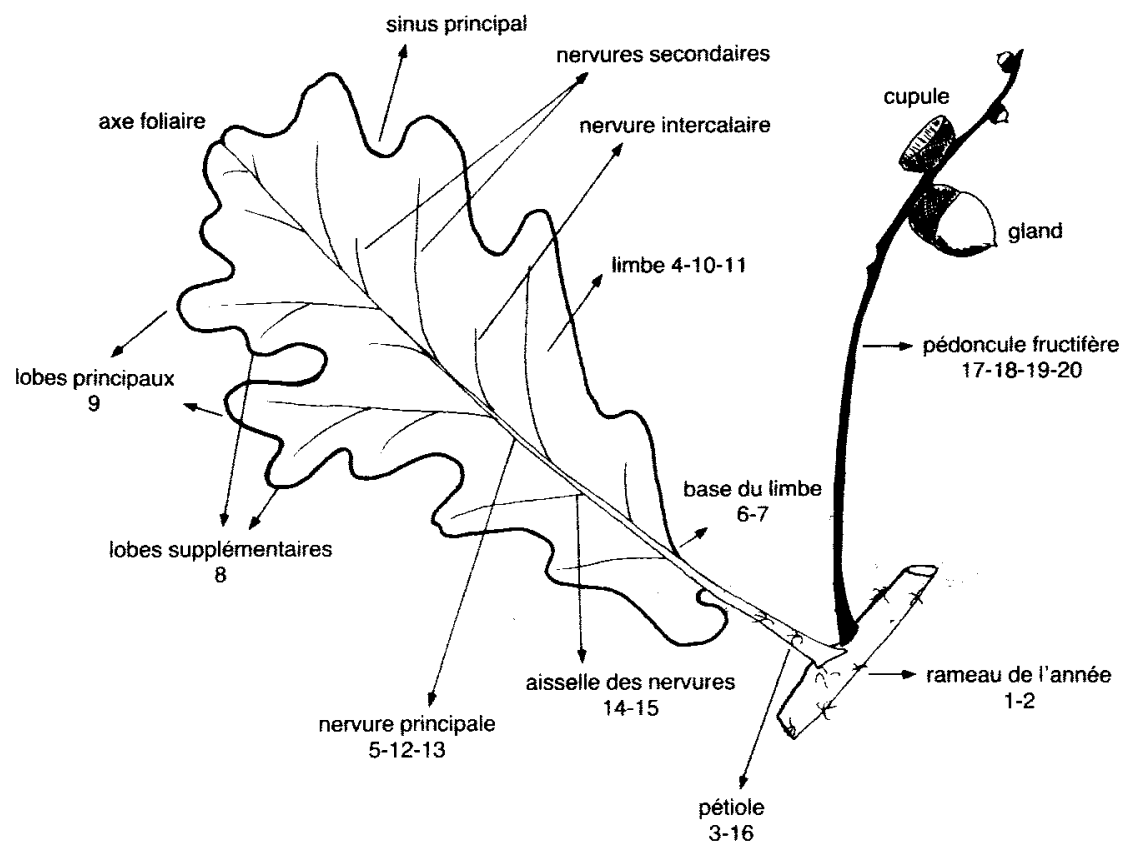

FIG. 1

Données récoltées sur l'échantillon (les numéros sont ceux des caractères taxonomiques).

(2) Ils sont déposés au Laboratoire de Botanique de l'E.N.I.T.E.F. 


\subsection{Caractères taxonomiques}

\subsection{Données prélevées sur l'échantillon}

Les caractères doivent être suffisamment discriminants pour rendre compte de la variabilité des arbres. Les meilleurs sont à la fois à peu près constants sur l'arbre, et variables entre les individus.

Ceux utilisés ici sont tirés de la littérature (Camus, 1939 ; Moggi \& Paoli, 1972 ; KISSLING, 1980a). Une étude préalable, portant sur 34 arbres et 30 caractères, a permis de choisir parmi ces derniers les plus fiables et les plus faciles à mesurer.

Les 20 caractères taxonomiques sont les suivants : (fig. 1).

\begin{tabular}{|c|c|}
\hline Rameau de l'année & $\begin{array}{l}1 \text { - Densité des poils tecteurs } \\
2 \text { - Longueur des poils }\end{array}$ \\
\hline Feuille & $\begin{array}{l}3 \text { - Couleur du pétiole } \\
4 \text { - Brillance de la face sup. du limbe } \\
5 \text { - Présence de nervures intercalaires } \\
6 \text { - Présence de } 1 / 2 \text { bases auriculées } \\
7 \text { - Présence de } 1 / 2 \text { bases aiguës } \\
8 \text { - Présence de lobes supplémentaires } \\
9 \text { - Forme de l'apex des lobes } \\
10 \text { - Densité des poils tecteurs à la face inf. du limbe } \\
11 \text { - Longueur des poils tecteurs à la face inf. du limbe } \\
12 \text { - Densité des poils tecteurs sur le pétiole ou de la nervure } \\
\text { principale } \\
13 \text { - Longueur des poils tecteurs sur le pétiole ou de la nervure } \\
\text { principale } \\
14 \text { - Densité des poils tecteurs à l'aisselle de la nervure principale } \\
15 \text { - Longueur des poils tecteurs à l'aisselle de la nervure principale } \\
16 \text { - Longueur moyenne des pétioles de } 10 \text { feuilles d'un rameau }\end{array}$ \\
\hline Pédoncule fructifère & $\begin{array}{l}17 \text { - Densité des poils tecteurs à mi-longueur } \\
18 \text { - Longueur des poils tecteurs à mi-longueur } \\
19 \text { - Moyenne des longueurs des pédoncules } \\
20 \text { - Ecart-type estimé de la longueur précédente (caractère non pris } \\
\text { en compte dans l'analyse multivariable) }\end{array}$ \\
\hline
\end{tabular}

\subsection{Protocole des mesures}

\subsection{Caractères métriques}

Les longueurs des pédoncules et des pétioles sont mesurées au millimètre près avec un réglet métallique. Le terme longueur du pédoncule désignera la distance de la base du pédoncule au niveau de l'insertion de la $1^{\text {*c }}$ cupule (ou de la cicatrice de celle-ci).

\subsection{Caractères de pilosité}

Ils sont parmi les plus distinctifs des 3 chênes. Une loupe binoculaire $35 \mathrm{X}$ est suffisante pour les mesures; on ne prend en compte que les poils tecteurs.

- Les densités sont appréciées par rapport à des échelles graphiques de référence (d'après Kissling, 1980a). Les 7 classes vont de $O$ (glabre) à $F$ (entièrement opaque). On note la classe moyenne observée sur l'ensemble de l'échantillon de l'arbre. 
- Les longueurs des poils sont appréhendées à l'aide de 3 classes d'une échelle graphique (d'après Kissling, 1977) : poils courts, moyens ou longs (les 3 pouvant coexister sur une même feuille).

\subsection{Autres caractères}

- 3: La couleur du pétiole est notée rouge, orange, jaune ou indéterminée (= verdâtre), d'après des couleurs de référence du Code International de Couleur des sols :

Rouge (R) : 7,5 R $(3 / 6,4 / 6,4 / 8)$ et $10 \mathrm{R}(4 / 6,5 / 6)$.

Jaune (j) : 5 Y $(7 / 8,8 / 4,8 / 6,8 / 8)$ et 2,5 Y $(7 / 6,7 / 8)$.

Orange $(\mathrm{O})$ : les intermédiaires entre ces 2 couleurs.

Indéterminée (I) : non identifiée dans le Code.

On ne considère que la couleur « dominante » trouvée dans l'échantillon.

- 4 : La feuille est notée subjectivement mate ou brillante.

- 5: Une nervure intercalaire irrigue un sinus principal, et est au moins aussi longue que la demi-distance : base de la nervure/base du sinus. On note en "présence » 3 nervures intercalaires ou plus en moyenne par feuille.

- 6-7-9: Les formes de la base du limbe et de l'apex des lobes sont appréciées à l'aide des échelles graphiques fig. $2 a$ et $2 b$. En ce qui concerne l'apex, on ne note que la forme "maximum " de l'échantillon, selon la relation d'ordre : $\mathrm{D}>\mathrm{A}>\mathrm{B}>\mathrm{C}$.

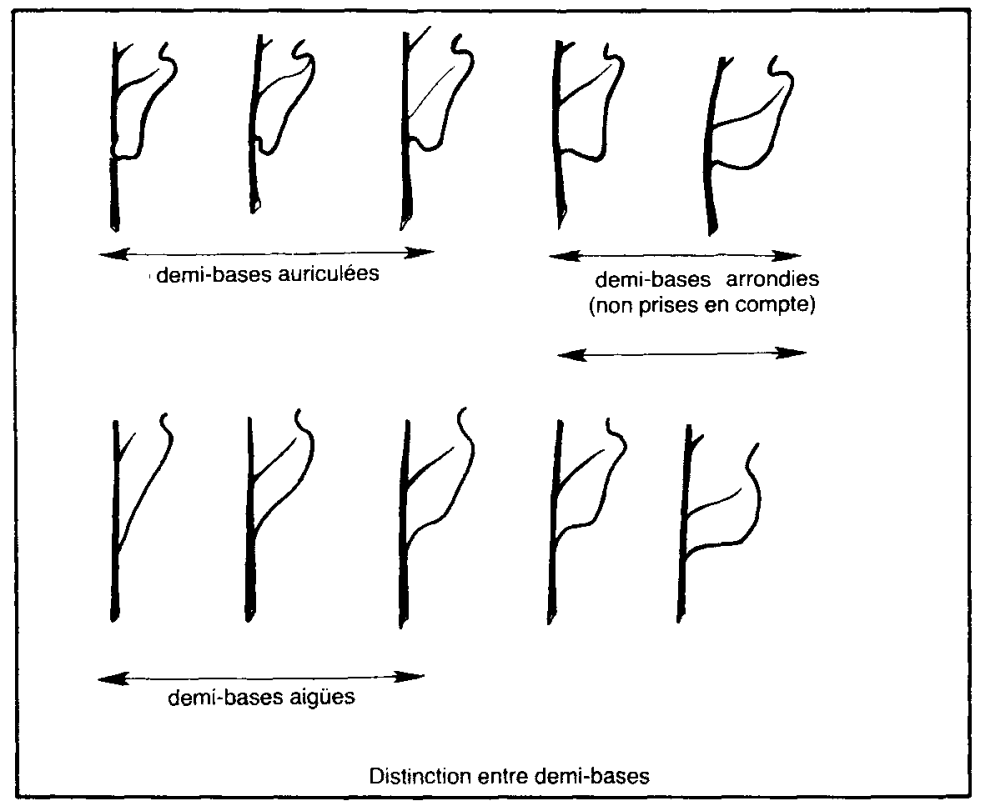

Fig. 2a

Forme de la base du limbe : échelle graphique (modifiée de MogG \& PAOLI, 1972). 


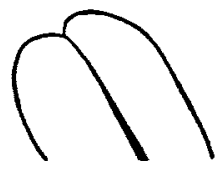

D

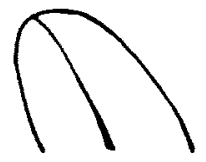

A

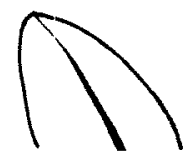

B

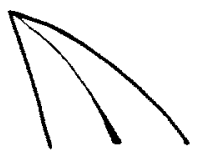

C

Fig. 2b

Forme de l'apex des lobes: tchelle graphique.

- 8: Un lobe supplémentaire est irrigué par une nervure d'ordre 3. Le caractère 8 correspond à au moins 2 lobes supplémentaires en moyenne par feuille.

On ne mesure essentiellement que des moyennes de l'échantillon récolté sur l'arbre. Les classes choisies, notamment pour les échelles graphiques, sont très larges, et il existe toujours une classe dont l'effectif est nettement supérieur aux effectifs des autres classes. On peut considérer la variation intra-arbre comme faible et négligeable. Quant aux caractères métriques, l'étude de leur variation fait l'objet de la seconde partie.

Au vu des histogrammes obtenus pour les longueurs moyennes dans l'échantillon total des 419 arbres, ces critères métriques continus ont été à leur tour sériés en classes, selon :

- longueur des pétioles : 8 classes, $\mathrm{mm}: 0-7,7-9,9-11,11-13,13-15,15-17,17-20,20-27$;

- longueur des pédoncules : 11 classes, $\mathrm{mm}: 0-6,6-8,8-10,10-12,12-14,14-16,16-18,18-20,20-22,22-40,40-80$.

Les 19 premiers caractères forment ainsi 82 classes binaires $(1 / 0)$.

\subsection{Traitements et résultats}

Le tableau des données : 419 arbres $\times 82$ classes binaires de caractères a été traité en analyse factorielle des correspondances (programme AFCE de l'E.N.I.T.E.F., version du 27-10-1982). Les résultats sont exploités à l'aide des projections dans les plans des 6 axes factoriels, et par le tableau diagonalisé des données.

\subsection{Projection graphique}

Les deux premiers facteurs constituent le meilleur plan de projection. La figure $3 a$ montre 3 ensembles d'arbres isolés par l'analyse. Tous les individus appartiennent à un «triangle " taxonomique, avec un fort regroupement des arbres vers les sommets.

La projection des classes de caractères confirme l'ordonnancement triangulaire, et montre une relation clinale très forte entre les diverses classes des caractères élémentaires (fig. 3b). 
FIG. 3

Projection graphique sur les axes factoriels $I$ et 2.

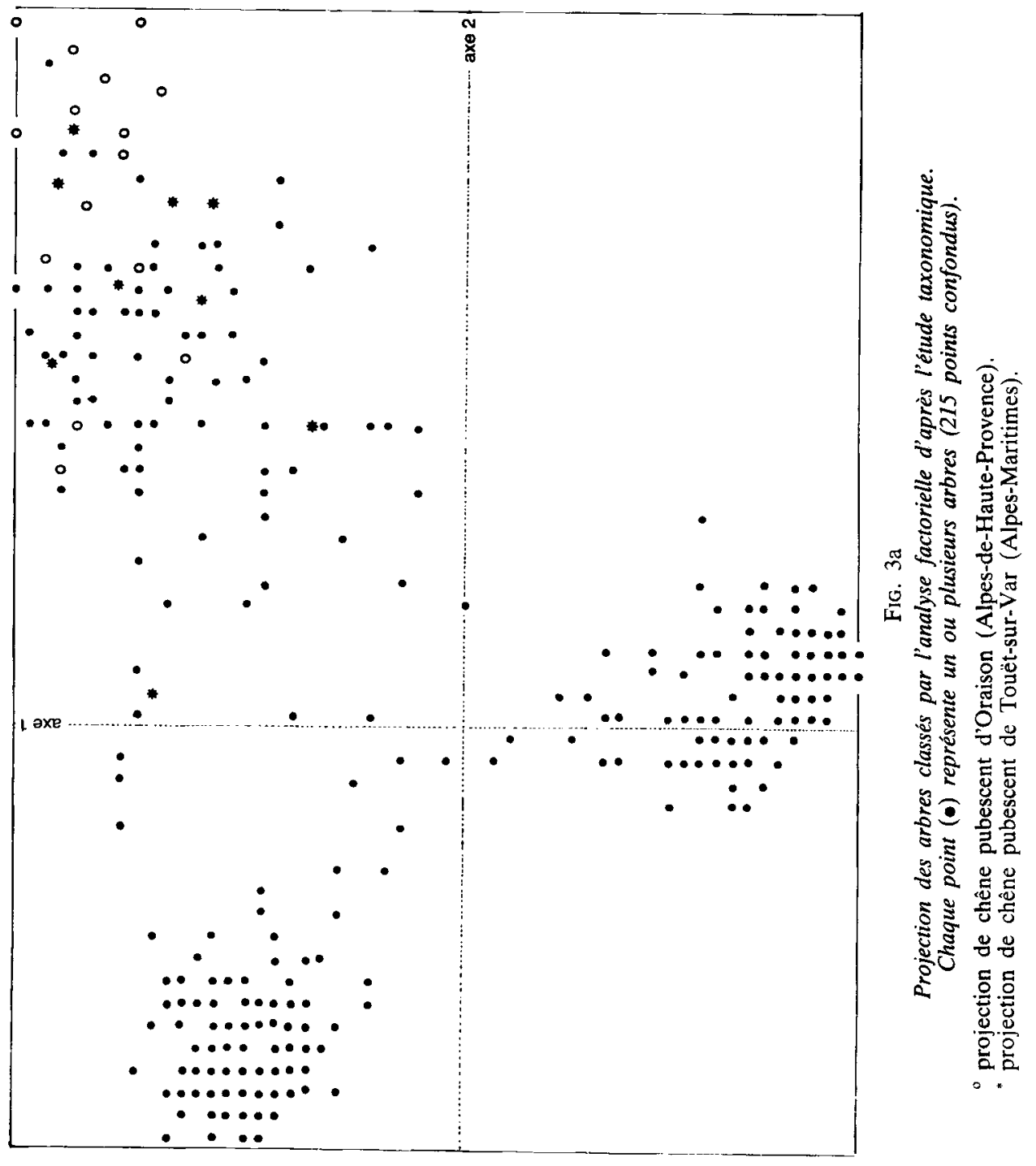



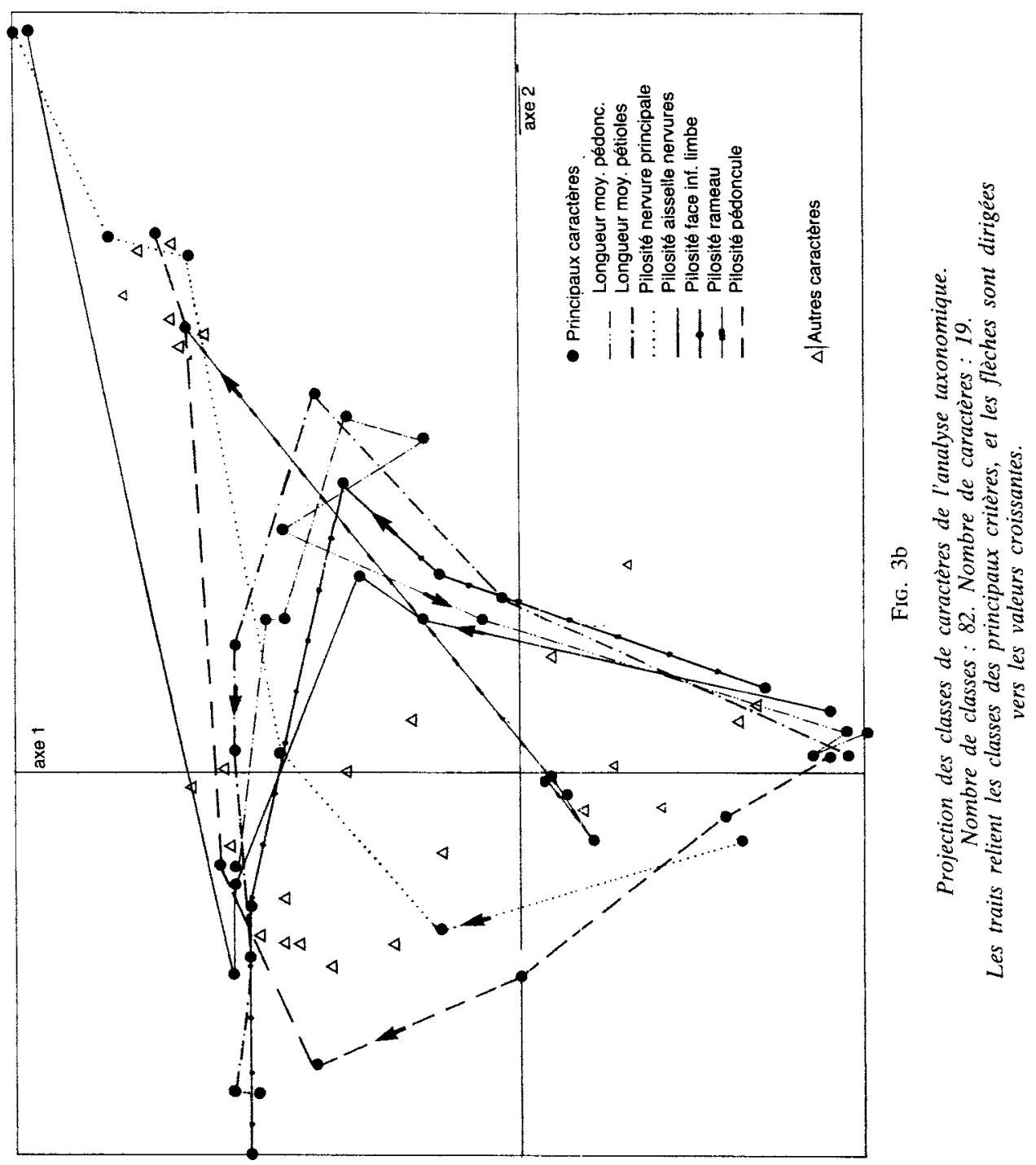
Les caractères groupés au voisinage de chaque sommet permettent de dresser un portrait-type des chênes qui s'en réclament.

Pôle 1 : La feuille est mate sur sa face supérieure.

Le pétiole, vert ou plus rarement rouge, est glabre et court (moins de $7 \mathrm{~mm}$ ).

Les feuilles possèdent plus de 3 nervures intercalaires, et sont auriculées. Le rameau de l'année, la face intérieure du limbe, la nervure principale, l'aisselle des nervures et le pédoncule fructifère sont glabres.

Enfin, le pédoncule fructifère est long (plus de $18 \mathrm{~mm}$ ).

Cette description est celle de Quercus robur (Kotschy, 1864 ; Camus, 1939 ; Moggi \& PaOLI, 1972 ; Kissling, 1980b).

Pôle 2 : Le rameau de l'année est glabre.

La feuille, luisante sur sa face supérieure, possède moins de 3 nervures intercalaires, et sa base n'est pas auriculée.

Le pétiole est long et jaune, glabre (plus de $12 \mathrm{~mm}$ de longueur moyenne).

La face inférieure du limbe présente des poils courts, appliqués, étoilés, en grand nombre: $C$ ou $D$.

L'aisselle de la nervure principale montre par contre des poils moyens ou longs, dressés, nombreux, $\mathrm{C}$ ou $\mathrm{D}$.

Le pédoncule fructifère, pileux (C ou D) de poils courts ou moyens, est court (moins de $6 \mathrm{~mm}$ ).

Cela correspond au profil de Quercus petraea.

Pôle 3: Il est beaucoup plus flou.

Le rameau de l'année est très pubescent $(E)$ masqué par la pilosité $(F)$.

La feuille, qui possède des lobes assez aigus, est luisante ; elle n'a ni oreillettes, ni nervures intercalaires. Son pétiole est long (plus de $11 \mathrm{~mm}$ ). La nervure principale, le pétiole et l'aisselle des nervures sont densement (E) pubescents de poils longs.

La face inférieure du limbe montre une densité moyenne (C) de poils longs uniquement.

Le pédoncule fructifère est court (moins de $8 \mathrm{~mm}$ ) et très pubescent ( $E$ ou F).

On peut y reconnaître Quercus pubescens.

Dès lors, la projection graphique des arbres (fig. 3a) s'interprète immédiatement : les deux ensembles denses correspondent aux chênes sessile et pédonculé, réunis par un isthme intermédiaire. La «nébuleuse » de droite regroupe des chênes pubescents.

Tous les arbres ne se reconnaissent évidemment pas dans les portraits-type précédents. Il existe une énorme variabilité, notamment dans l'ensemble pubescent, pouvant aller jusqu'à une absence totale de poils tecteurs sur le rameau et la feuille !

Le regroupement important des chênes vers les pôles autorise cependant à donner à ces ensembles les noms spécifiques : l'Espèce, loin d'être l'incarnation d'un type fixe, prend au contraire en compte la variabilité d'une population.

C'est une notion essentiellement pratique, et donc vulgarisatrice.

Il ne peut être donné aux axes factoriels de définition précise. Tout au plus peuton dire, à l'examen du poids des classes de caractères, que l'axe 1 prend beaucoup en 
compte les longueurs des pétioles et des pédoncules, alors que l'axe 2, qui seul sépare les 3 chênes, est principalement lié aux caractères de pilosité.

\subsection{Tableau diagonalisé}

Le tableau des données $(419 \times 82)$ présente les arbres et les caractères dans l'ordre de leur numérotation.

En permutant de manière judicieuse l'ordre des lignes (caractères) et des colonnes (arbres), on peut regrouper préférentiellement les informations (caractères codés 1) au voisinage de la diagonale principale. On obtient ainsi un tableau diagonalisé (fig. 4).

Les 3 espèces apparaissent nettement, airısi que la distribution de chaque classe de caractère dans l'ensemble des arbres. L'examen de l'étalement de tous les caractères permet de tracer des limites entre les espèces.

Ces limites sont généralement bien accusées; seuls 12 individus entre les chênes sessile et pédonculé ont été qualifiés $d^{\prime}$ ' intermédiaires ", l'ensemble des caractères décrits ne permettant pas de les rattacher à une espèce plutôt qu'à l'autre.

\subsection{Sur quelques chênes méditerranéens}

Le chêne pubescent, espèce méditerranéenne, est en transgression édaphique en Berry. Il était donc intéressant de comparer les individus berrichons à leurs homologues méridionaux.

Vingt-cinq chênes à feuilles caduques ont été échantillonnés dans deux taillis vieillis, l'un à Oraison (Alpes de Haute-Provence), l'autre à Touët-sur-Var (AlpesMaritimes).

Ces arbres ont été décrits et dépouillés dans les mêmes conditions que les 419 autres chênes. La projection graphique dans le plan factoriel $(1,2)$ montre exactement les mêmes regroupements de chênes berrichons, mais les chênes méditerranéens sont dans leur majorité plus proches du pôle pubescent que tous les autres. Leurs caractères, notamment de pilosité, sont plus prononcés (fig. 3a).

\subsection{Interprétation et modèles génétiques}

L'étude n'a porté que sur des corrélations entre caractères morphologiques. Pour interpréter plus avant le triangle taxonomique, un modèle théorique reliant ces caractères et leur support génétique est nécessaire.

Pour ANDERSon, 1949, et Kissling, 1980b, l'introgression (hybridation suivie de back-cross) entre plusieurs espèces produit un « réseau de corrélations », dû au linkage des caractères polygéniques tempéré par le crossing-over ${ }^{(3)}$.

Sous réserve de certaines hypothèses génétiques, les individus F2 issus de l'introgression entre 3 espèces ont des caractères intermédiaires entre ceux des espèces parentales. Ils peuvent être représentés par un ensemble de points situés à l'intérieur d'un triangle dont les sommets seraient les parents. Dans les générations suivantes, le réseau est fragmenté, mais non dénaturé.

(3) D'autres auteurs estiment que les liaisons entre caractère sont plutôt d'origine pléiotropique. 

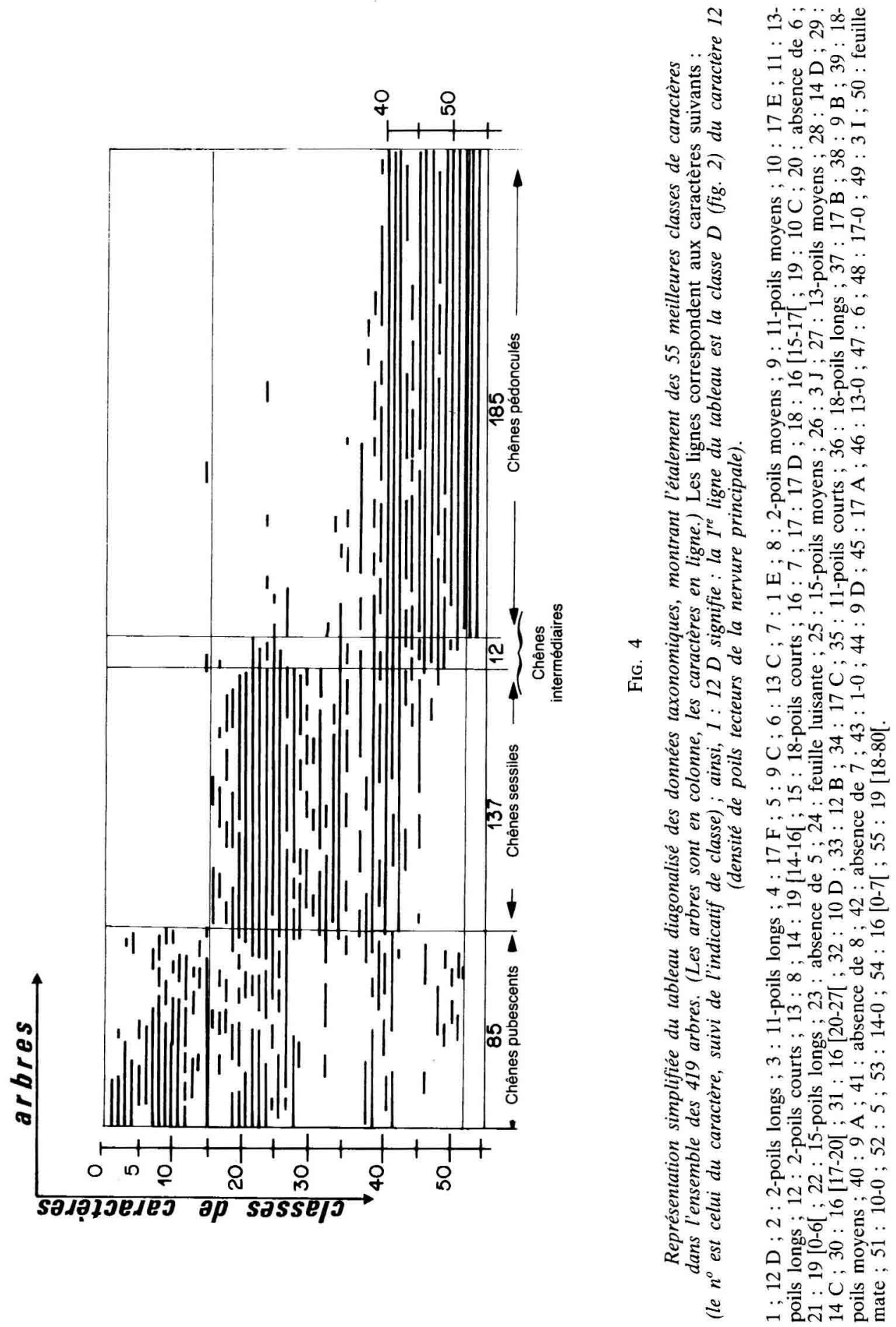

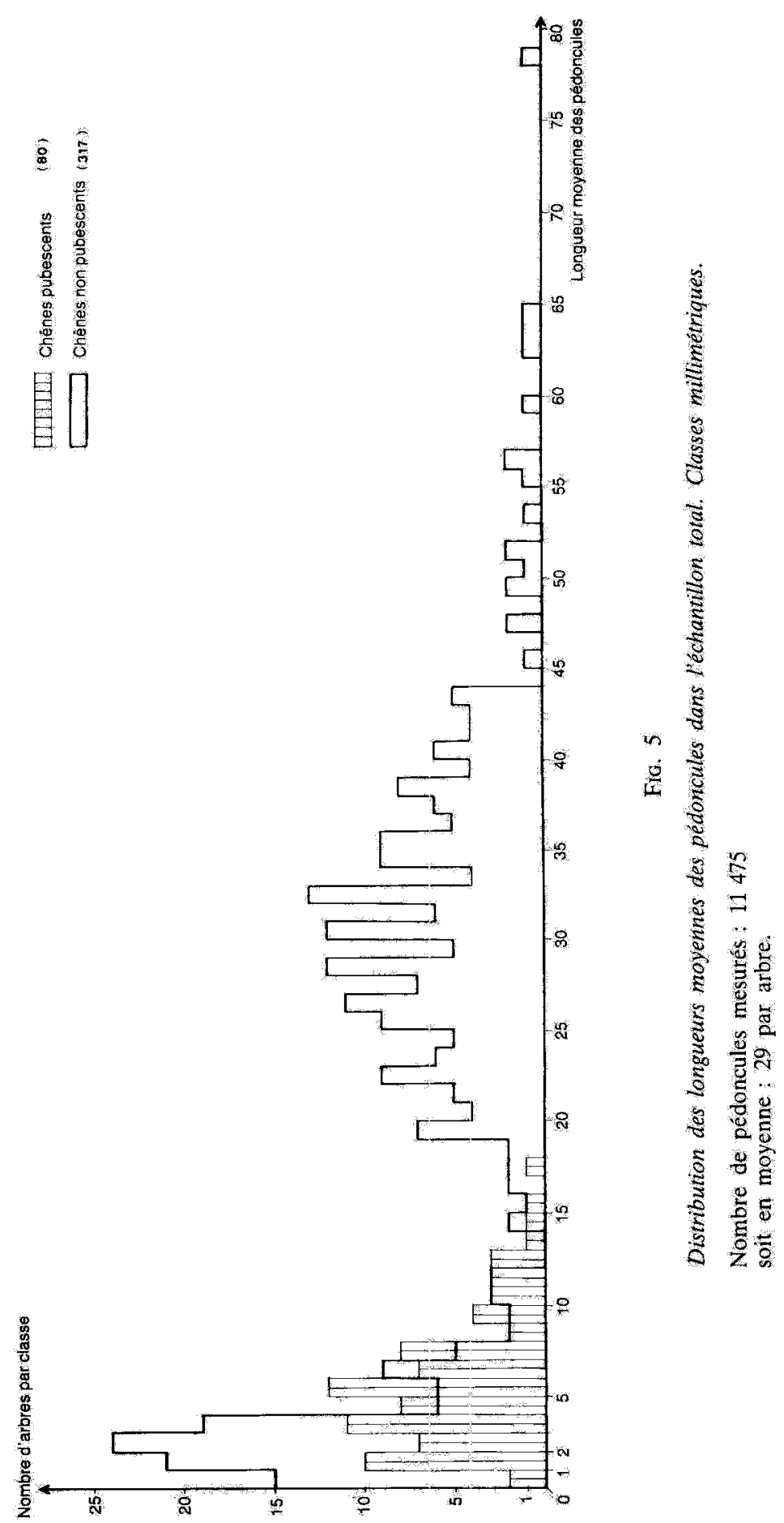
L'analyse factorielle calculant les corrélations entre les caractères différentiels, la projection graphique $(1,2)$ représente exactement le réseau théorique de KissLiNG.

Certaines hypothèses simplificatrices de l'auteur (caractères commandés par des polygènes à effets additifs, équiprobabilité de fécondation) s'accommodent mal du fort regroupement des chênes en Berry, et de la rareté des intermédiaires.

Pour expliquer ce fait, on peut évoquer en particulier :

- la dominance de certains gènes : voir $\$ 2.113$. la longueur du pédoncule;

- le pas variable des classes de caractères métriques (axe 1) privilégiant les longueurs intermédiaires. Les distributions dans l'échantillon (fig. 5) montrent que des pas équidistants isolent aussi nettement les chênes sessile et pédonculé ;

- la non équiprobabilité de fécondation entre les différentes formes;

- la sous-représentation de certains milieux écologiques. Cela ne peut être évoqué que pour la transition pédonculé-pubescent (voir $\$$ écologie).

Le réseau de corrélations permet enfin d'interpréter le triangle taxonomique comme conséquences d'un patrimoine génétique commun aux 3 chênes européens, avec une nette polarisation vers des formes distinctes et différenciées.

\subsection{Autres approches taxonomiques}

Des critères autres que morphologiques ont été utilisés par certains auteurs pour affiner la systématique des chênes.

\subsection{Les critères caryologiques}

Ils ne sont pas distinctifs : la garniture chromosomique est la même $(n=12)$, et l'étude fine des chromosomes est délicate : ceux-ci sont petits, se prêtent mal aux analyses classiques, et la présence constante de tanins vient polluer les préparations (Wigston, 1973).

\subsection{Une faible viabilité pollinique}

Elle est assez souvent le signe d'un statut d'hybride. Olsson, 1975 et Rushton, 1978 constatent une viabilité pollinique plus réduite chez leurs formes intermédiaires.

\subsection{Critères biochimiques}

L'examen des substances flavoniques est décevant (LeBreton, 1976) mais Olsson, 1976, met en évidence un groupe de péroxydases distinctives de Quercus robur et Quercus petraea.

\subsection{Les hybridations interspécifiques artificielles}

Elles sont peu probantes Dengler, 1941 ; Pyatnitski, 1960 (in Gardiner, 1970) ; Jovanovic, 1973 ; Rushton, 1977, rapportent des taux moyens de fécondations robur $\times$ petraea et robur $\times$ pubescens variant entre 0 et 2 p. 100 (contre 30 à 50 p. 100 pour les fécondations intraspécifiques). De plus, les glands formés sont extrêmement fragiles : aucun hybride viable n'a été obtenu à ce jour ! 
Ceci mériterait cependant d'être vérifié compte tenu du peu de connaissances actuelles sur la maitrise de la biologie florale des chênes.

1.65. Les paramètres technologiques des bois, étudiés par DerET-VARCin (1983), isolent bien les chênes sessile et pédonculé.

Le polymorphisme de nombreux chênes américains a aussi été attribué à l'introgression. Les études biochimiques multivariables viennent corroborer et affiner les résultats des analyses morphologiques (KNOPS \& JENSEN, 1980).

\section{Variations}

Les résultats de l'analyse taxonomique multivariable rendent possible l'interprétation de la variation de quelques caractères.

\subsection{Caractères métriques}

Les longueurs des pétioles et des pédoncules ont fait l'objet de mesures millimétriques sur chaque arbre :

- variation individuelle, grâce à quelques arbres suréchantillonnés ;

- variation dans deux populations sympatiques, les placettes 02 et 04 , portant des peuplements purs respectivement sessile et pédonculé ;

- variation dans l'échantillon total (419 arbres) à l'aide des longueurs moyennes par arbre.

\subsection{Longueur des pédoncules fructifères}

\subsection{Variation individuelle}

Chez tous les individus, la distribution est monomodale et dissymétrique (fig. 6). Les courbes bimodales obtenues par Pellecuer, 1976, pourraient résulter d'une insuffisance statistique des échantillons. Ces courbes en cloches suggèrent un contrôle polygénique du caractère (hypothèse envisagée par OLSSON, 1976).

Enfin, leur dissymétrie fait penser à une distribution log-normale. C'est le cas chez le chêne pédonculé ; il faudrait des mesures plus précises dans la classe $[0,1[\mathrm{~mm}$ pour le vérifier aussi chez le sessile.

\subsection{Variation d'une population}

Dans les placettes 02 et 04 , on a fait la somme des fréquences relatives des classes. Ici aussi, les courbes sont monomodales dissymétriques (fig. 7). On observe de plus un léger recouvrement dans les classes de trarısition (5 à $15 \mathrm{~mm}$ ), même pour des peuplements purs.

Pour la placette 04, le passage aux coordonnées semi-logarithmiques permet d'obtenir une distribution normale. 


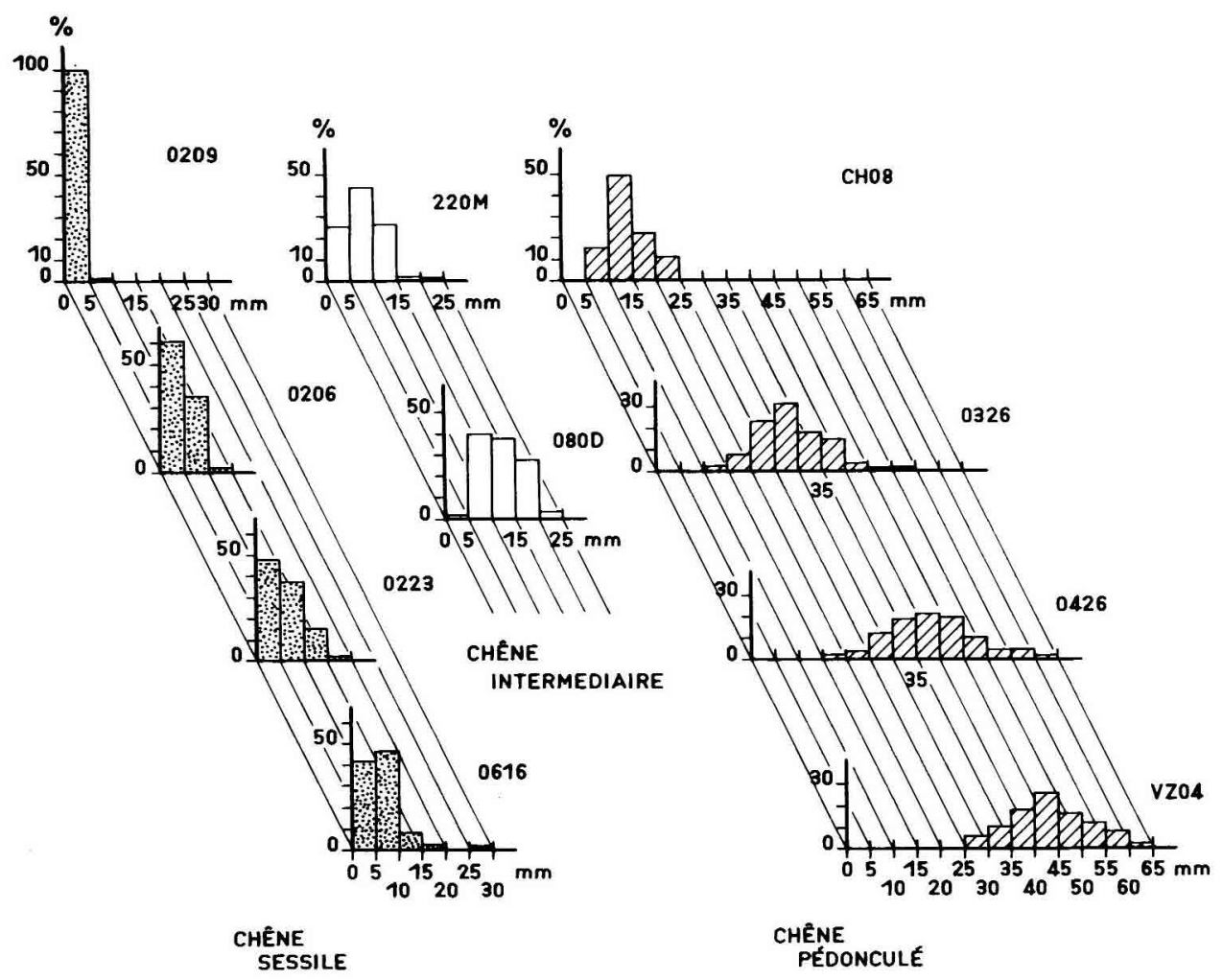

FIG. 6

Exemples de la variation de la longueur des pédoncules sur différents arbres de l'échantillon.

Abscisse : longueur des pédoncules de l'arbre (classe : $5 \mathrm{~mm}$ ).

Ordonnées : \% par classe.

$0209: n^{\circ}$ de l'arbre.

\subsection{Variation dans l'échantillon}

On utilise la longueur moyenne par arbre $\overline{\mathrm{L}}_{\mathrm{d}}$. Cette grandeur isole 2 ensembles (chênes pubescents exceptés) qui correspondent à quelques pour cent près aux chênes sessile et pédonculé : le nom français de ces espèces est ainsi justifié (fig. 5).

Au-delà de $\bar{L}_{d}=17 \mathrm{~mm}$, tous les chênes communs sont des pédonculés. En-deçà de $\overline{\mathrm{L}}_{\mathrm{d}}=8 \mathrm{~mm}, 96$ p. 100 sont des sessiles. Entre ces deux seuils, on ne peut dire a priori l'espèce. Les intermédiaires se distribuent dans les classes $[5,15[\mathrm{~mm}$.

Il apparaît d'autre part que chez les chênes pédonculés, longueur moyenne $\overline{\mathrm{L}}_{\mathrm{d}}$ et coefficient de variation $\mathrm{C}_{\mathrm{v}}$ sont indépendants.

Ces observations : indépendance du coefficient de variation, et distribution lognormale, font tenir pour très vraisemblable que les gènes contrôlant la longueur des pédoncules du chêne pédonculé ont des effets multiplicatifs. La dissymétrie des courbes serait ainsi due à une dominance partielle des gènes dont les effets sont les plus faibles. 


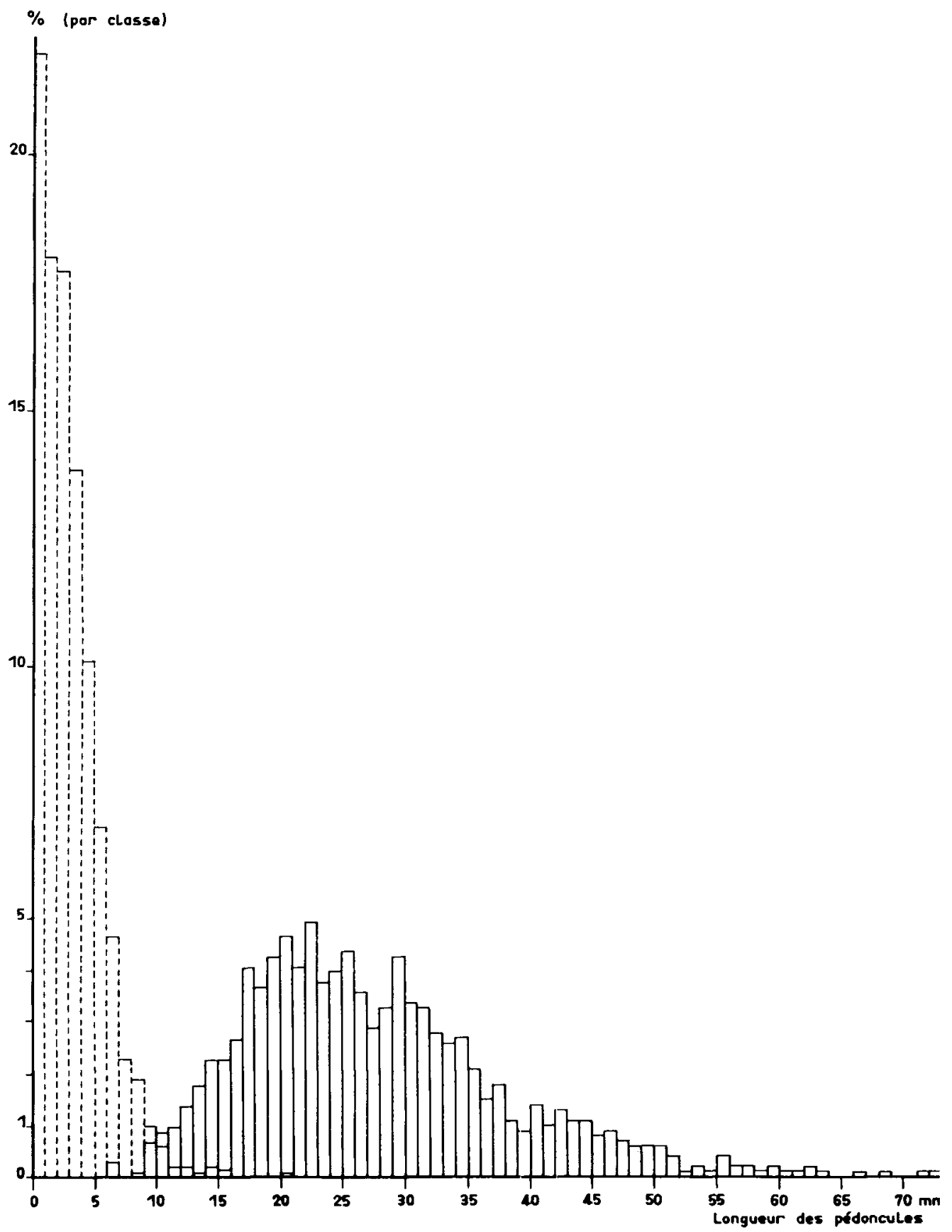

Fig. 7

Histogramme des fréquences des iongueurs de pédoncule.

dans une placette (02) de chêne pédonculé ( 26 arţres et 2259 pédoncules).

dans une placette (04) de chêne sessile ( 30 arbres et 2022 pédoncules). 


\subsection{Longueur des pétioles}

\subsection{Variation individuelle}

Chez tous les individus, la distribution est monomodale. Les courbes sont moins nettes que celles obtenues pour les pédoncules. Le mode d'échantillonnage est peut-être en cause : les feuilles d'un rameau n'ont certainement pas des longueurs de pétiole indépendantes.

\subsection{Variation dans une population}

A lintérieur des placettes monospécifiques 02 et 04 , la distribution de la longueur des pétioles de tous les arbres est normale. Shutilov, 1968, observe de telles distributions aussi chez les chênes pubescents du Caucase.

\subsection{Variation dans l'échantillon}

- Longueur moyenne des pétioles $\overrightarrow{\mathrm{L}}_{\mathrm{t}}$ et coefficient de variation ne présentent aucune liaison particulière.

- La courbe obtenue avec $\bar{L}_{\mathrm{t}}$ est semblable à la figure 5 : Chênes pubescents mis à part, il y a une bonne séparation sessiles/pédonculés.

Au-delà de $\bar{L}_{t}=14 \mathrm{~mm}$, tous les non-pubescents sont des sessiles.

En-deçà de $\bar{L}_{t}=7 \mathrm{~mm}$, tous les chênes sont des pédonculés.

- On peut raisonnablement penser, au vu des distributions normales, que le contrôle de la longueur du pédoncule est réalisé par un polygène à effets additifs.

\subsection{Variations géographiques}

Les comparaisons ponctuelles avec d'autres travaux sont difficiles du fait de la diversité des variables taxonomiques employées, et des traitements choisis par les auteurs. On se bornera ici à comparer les populations avec l'excellent critère de longueur moyenne des pédoncules.

\subsection{Les chênes en forêt de Morimond (Haute-Marne) (Pellecuer, 1976)}

Les chênes pubescents ne sont pas signalés à Morimond, et ont donc été exclus des courbes en Berry. Les classes choisies par l'auteur sont centimétriques, excepté entre 0 et $1 \mathrm{~cm}$.

Les conditions de deux études sont très différentes (climat, sols, traitement sylvicole), mais les deux ensembles, sessiles et pédonculés, sont bien tranchés. Il ne paraît pas y avoir une grande quantité d'" intermédiaires» (fig. 8a).

\subsection{Les chênes de la Vallée de l'Ognon (Jura) (Mourey, 1978)}

Des chênes pubescents sont signalés par l'auteur. Les courbes de distribution de $\overline{\mathrm{L}}_{\mathrm{d}}$ en Jura et Berry sont très semblables, le premier pic correspond aux chênes sessile et pubescent, le second aux pédonculés (fig. 8b). 
FIG. 8

Fréquence des chênes par classe de longueur moyenne des pédoncules en Jura, Haute-Marne, Suède et Berry.
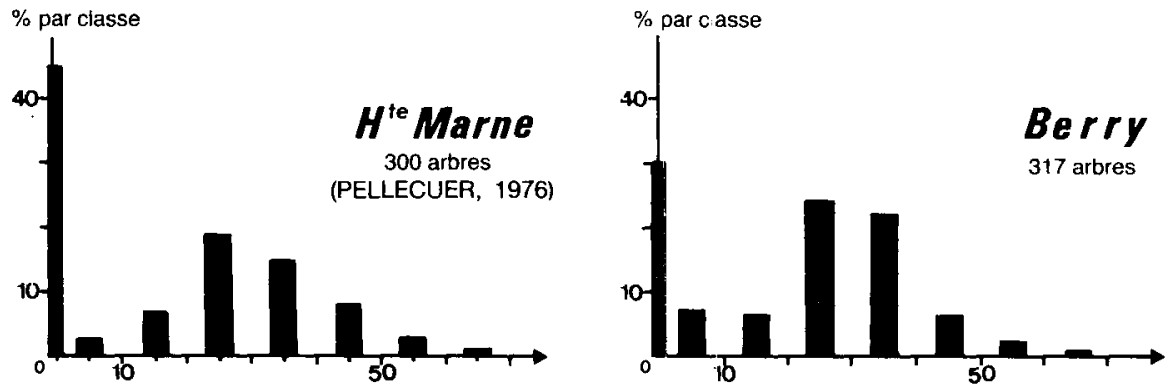

FiG. $8 \mathrm{a}$

Comparaisons des distributions en Haute-Marne et Berry. Classes : $10 \mathrm{~mm}(5 \mathrm{~mm}$ entre 0 et 1$)$.
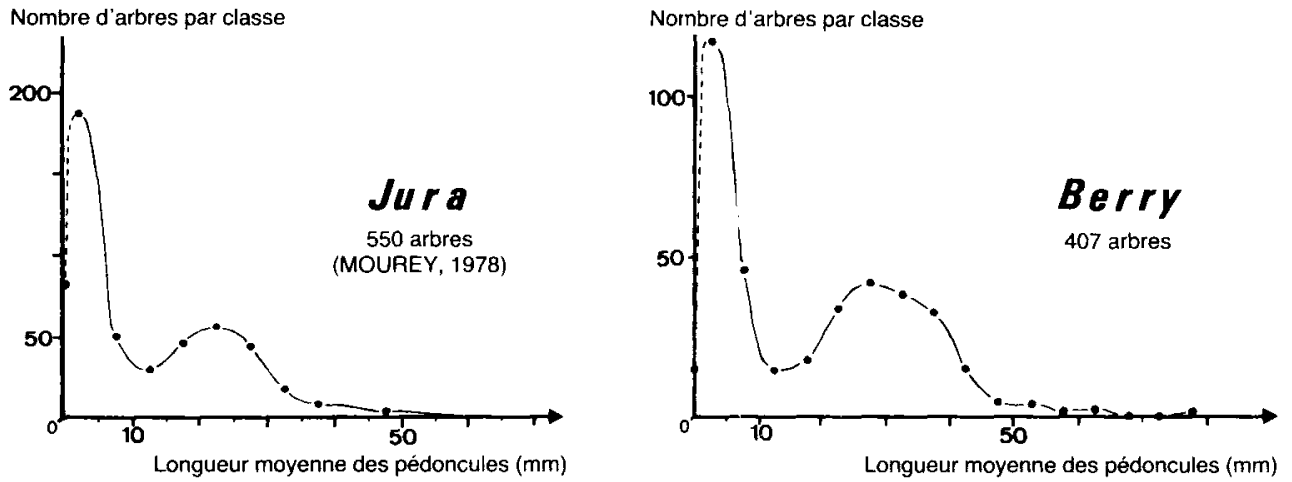

FIG. $8 b$

Comparaison des distributions en Jura et Berry.
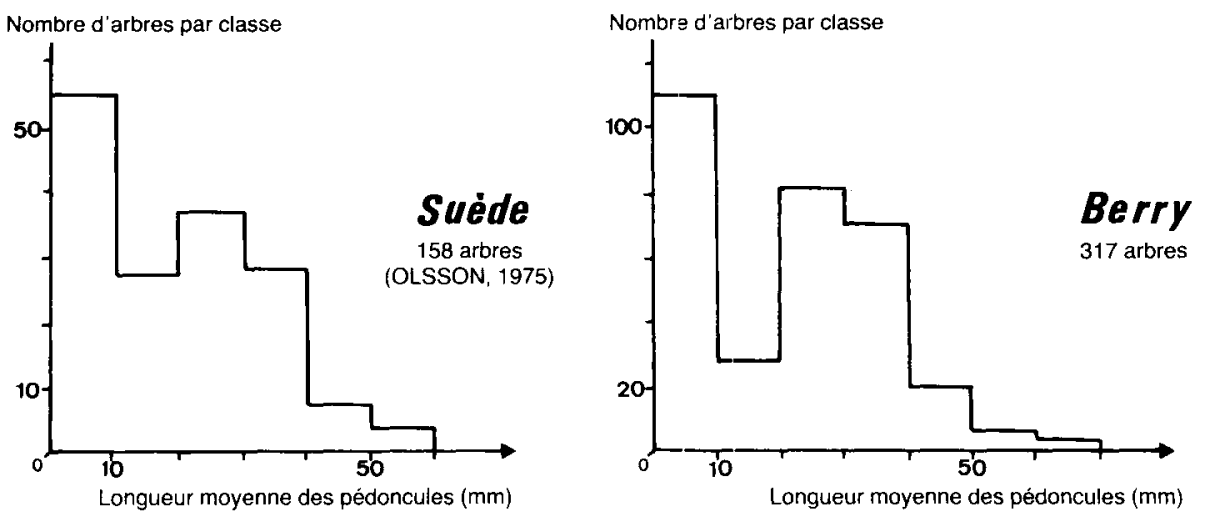

FIG. 8c

Comparaison des distributions en Suède et Berry. Classes : $10 \mathrm{~mm}$. 


\subsection{Les chênes de Suède (OLsson, 1975) (fig. 8c)}

La distribution de cette figure a été tracée à partir de schémas donnés par l'auteur. Elle présente, par rapport à celles étudiées en Berry, une hypertrophie de la classe $[10,20[\mathrm{~mm}$ où sont trouvés intermédiaires et pédonculés berrichons.

Si le caractère "longueur moyenne du pédoncule fructifère " a la même signification partout (ce qui serait en accord avec le réseau de corrélations), la distinction sessile/pédonculé semble pouvoir s'appliquer à une bonne partie de la France, alors qu'elle paraît comparativement moins nette en Suède où le chêne sessile est en limite géographique de son aire de distribution.

\subsection{Conclusion de l'étude botanique}

En Berry, les trois principaux chênes européens : Quercus robur, Quercus petraea et Quercus pubescens existent de manière caractéristique.

Tous les arbres s'inscrivent dans un triangle taxonomique dont les angles sont des espèces-type. Les chênes pubescents méditerranéens sont plus proches du pôle pubescent que leurs homologues berrichons qui présentent une forte variabilité. Aucun chêne indigène observé ne présente de caractères étrangers aux trois espèces.

Cependant la prise en compte d'un grand nombre d'arbres et de caractères différentiels fiables ne permet pas de doute sur la réalité mais aussi la rareté des chênes intermédiaires.

La séparation botanique classique est ainsi confirmée : l'espèce est une entité statistique qui prend en compte la variabilité d'une population d'arbres interféconds. Cette distinction est nécessaire et suffisante pour la gestion forestière.

On a vérifié de plus que la prise en compte d'un ou deux caractères suffit à reconnaître - mais non pas établir - les espèces. C'est ainsi que la confrontation des distributions de la longueur moyenne des pédoncules en Haute-Marne, Jura et Berry, montre la nette séparation des chênes sessile et pédonculé, alors que les « intermédiaires » semblent plus fréquents en Suède.

\section{Ecologie}

\subsection{Etude phytoécologique}

Une étude complète du milieu naturel doit prendre en compte les variations climatiques, stationnelles et biotiques.

En ce qui concerne les premières, il faudrait envisager les micro-variations de température et de pluviométrie, à l'intérieur de la forêt, voire de la placette. Il s'agit d'un travail important, dépassant le cadre de cette étude.

Les variables biotiques ont été considérées comme identiques a priori partout.

Dans un milieu donné, les ensembles floristiques représentent une synthèse particulièrement intéressante de toutes ces influences, et permettent un classement relatif des placettes selon les principaux facteurs du milieu. 
L'étude phytoécologique réalisée ici a pris en compte un nombre réduit d'espèces végétales ayant une signification très importante en Région Centre.

La diagonalisation du tableau des données (78 espèces $\times 32$ placettes) a permis le regroupement des placettes semblables en stations phytoécologiques. Des descriptions pédologiques ont vérifié la validité de ce classement ${ }^{(4)}$.

Les grands milieux échantillonnés sont les suivants : alluvial, calcaire, neutre, xéroacidophile et hydromorphe. Dans chacun d'entre eux, ont été analysées la répartition des espèces de chênes (autoécologie) et celle des peuplements (synécologie).

\subsection{Autoécologie}

Lors de l'analyse taxonomique, les chênes ont été regroupés en pubescent, sessile, pédonculé et intermédiaire.

Dans chaque grand milieu écologique, le pourcentage de chaque groupe a été calculé (fig. 9) :
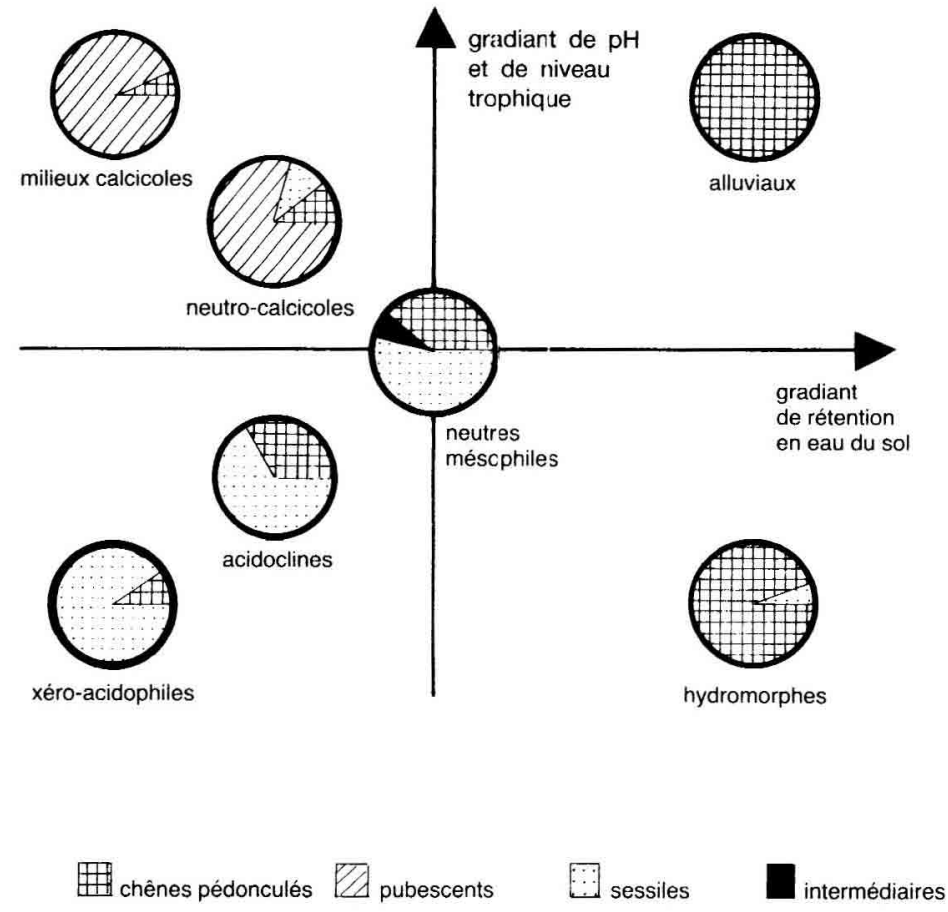

Fig. 9

Répartition écologique des chênes en Berry (Sologne exceptée) en \% par espèce.

(4) L'étude phytoécologique et les descriptions pédologiques sont détaillées dans le mémoire E.N.I.T.E.F. de Sigaud, 1984. 
- le chêne pubescent est cantonné en Berry aux calcaires superficiels (à moins de $35 \mathrm{~cm}$ de profondeur). Son comportement est xérothermophile;

- le chêne pédonculé, bien que présent partout à l'état diffus, a une préférence marquée pour les milieux humides (son optimum est en milieu alluvial) : c'est le plus hygrophile ;

- le chène sessile occupe les terrains xéro-acidophiles à neutres mésophiles. Sa hauteur dominante maximale est trouvée en milieu acidocline. Il apparaît surtout tolérer la sécheresse et l'acidité ;

- les intermédiaires sessile $\mathrm{x}$ pédonculé se trouvent uniquement dans des terrains neutres mésophiles, et en peuplements mélangés.

Ces exigences écologiques correspondent à celles mentionnées dans la littérature (Mathieu, 1877 ; Camus, 1938-1939 ; Rushton, 1979 ; Kissling, 1983 ; Duhamel, 1984).

\subsection{Synécologie}

L'accent va être mis désormais sur la variation des populations des placettes dans divers milieux écologiques.

Le triangle taxonomique formé par la projection des individus dans le plan factoriel $(1,2)$ est un instrument privilégié puisqu'il prend en compte tous les caractères taxonomiques. Pour visualiser la projection d'une placette ou d'un ensemble de placettes, il suffit de ne présenter dans ce triangle que les arbres de la (ou des) placette(s) en question.

\subsection{Les milieux alluviaux (fig. 10)}

Ils sont caractérisés par une alimentation constante en eau (par nappe alluviale) et par une richesse minérale élevée. Le chêne pédonculé y est exclusif. A mesure que le caractère alluvial disparaît, le centre de gravité des populations se déplace sur l'axe 1 , et la variance des populations augmente ; les caractères des arbres sont de moins en moins typés lorque l'on s'éloigne de l'optimum écologique. Mourey, 1978, observait en Jura une corrélation identique entre la longueur du pédoncule des arbres et l'humidité du milieu.

\subsection{Les milieux calcaires (fig. 11)}

Ils portent typiquement des chênaies pubescentes. Ces chênaies ont des caractères d'autant plus marqués que le calcaire est moins profond, ce qui revient à dire comme précédemment que la morphologie des populations varie de manière clinale avec le facteur déterminant du milieu.

Les chênaies-charmaies neutro-calcicoles (Quercion pubescenti petraeae de BraunBlanquet, 1931) font la transition entre les chênes sessile et pubescent, et on observe tous les stades intermédiaires de ces deux espèces.

Le facteur calcaire correspond en fait à un gradient thermique. Dans cette optique, les chênaies méditerranéennes extrapolent la corrélation observée ici entre la température et l'intensité des caractères. StEfanoff \& Kostov, 1962, observaient le même phénomène dans les chênaies pubescentes bulgares. 
FIG. 0

Structure taxonomique de's chênaies alluviales.

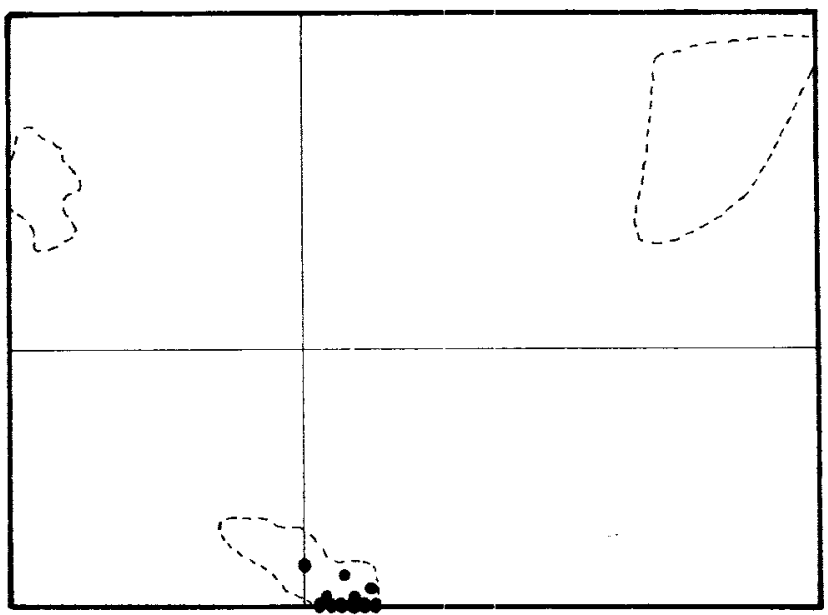

FIG. 10a

Chênaie-frênaie.

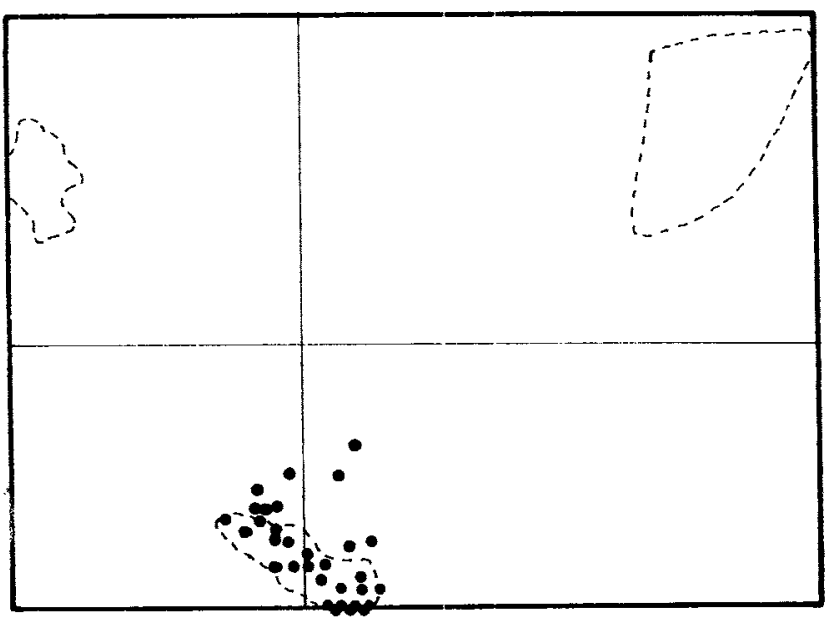

FIG. 10b

Chênaie pédlonculée. 
Fig. 11

Structure taxonomique des chênaies calcaires.

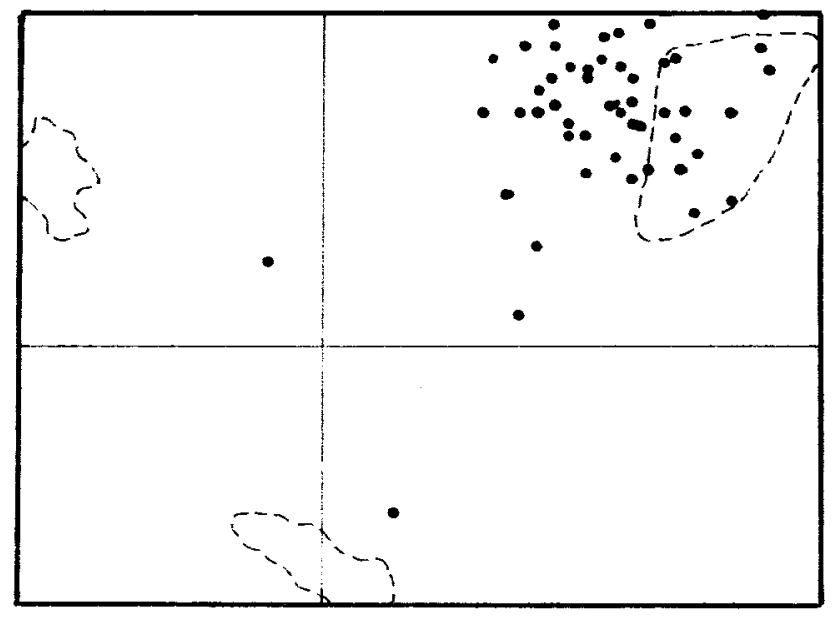

FIG. 11a

Chênaie pubescente xéro-thermophile.

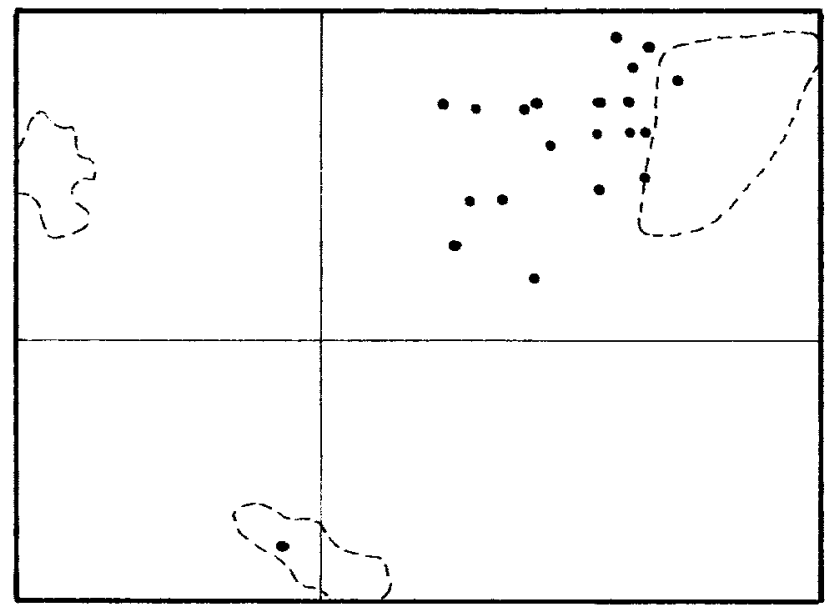

FIG. $11 \mathrm{~b}$

Chênaie-charmaie calcicole. 


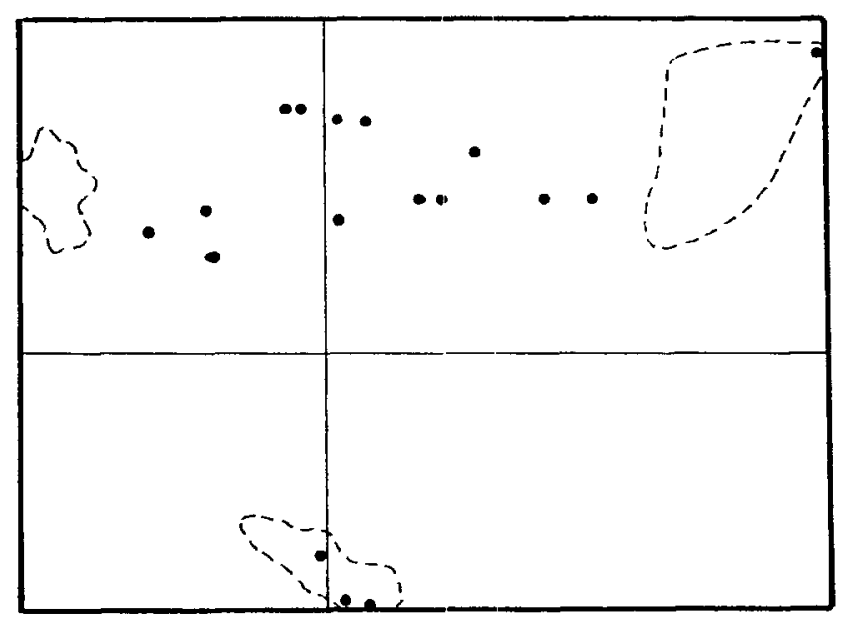

FIG. 11c

Chênaie-charmaie neutro-calcicole.

\subsection{Les milieux neutres mésophiles (fig. 12)}

Ce sont des milieux de transition entre des terrains mieux drainés et souvent plus acides (à chêne sessile), et des terrains topographiquement plus bas et mouilleux, préférés par le pédonculé.

Aucun chêne pubescent n'a été observé dans ces milieux.

Le point marquant est le fait que les individus intermédiaires sessile $\times$ pédonculé ne sont trouvés qu'ici, en peuplements mélangés, et toujours en faible nombre.

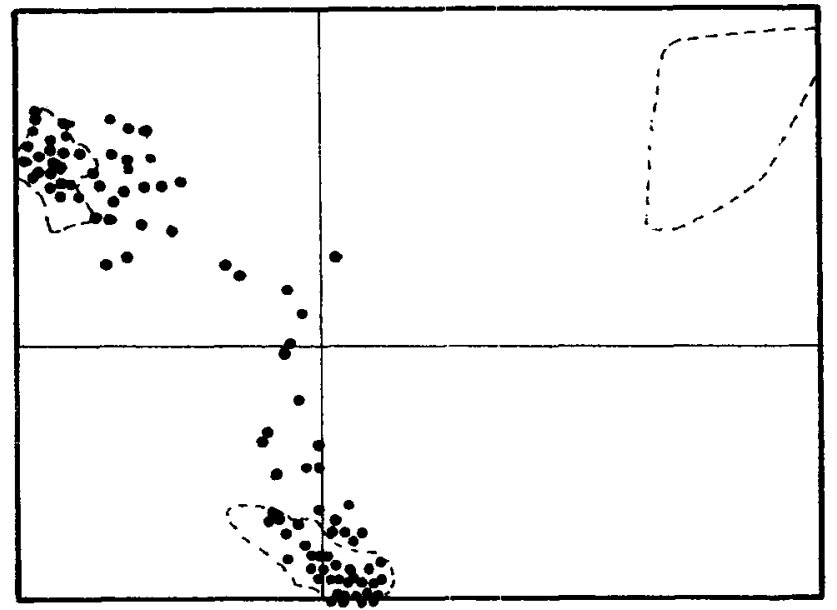

Fig. 12

Structure taxonomique des chênaies neutrophiles. 
La grande variation morphologique des arbres dans ces milieux est aussi attestée par Krahl-Urban, 1959 (Allemagne) ; Olsson, 1975 (Suède) ; Rushton, 1979 (Angleterre et Pays de Galles); Kissling, 1983 (Suisse).

\subsection{Les milieux xéro-acidophiles (fig. 13)}

Sont regroupées sous cette appellation les stations acidoclines et acidophiles sèches. Les peuplements sont constitués de chêne sessile pur très caractérisé, à l'exception des placettes solognotes où le chêne pédonculé est quasi exclusif.

La présence du chêne pédonculé dans ces chênaies peut étonner, mais le problème se pose à l'échelle de la Sologne entière. Il faut faire intervenir des facteurs anthropiques, la région ayant pendant très longtemps été cultivée en seigle, puis reboisée naturellement ou artificiellement.

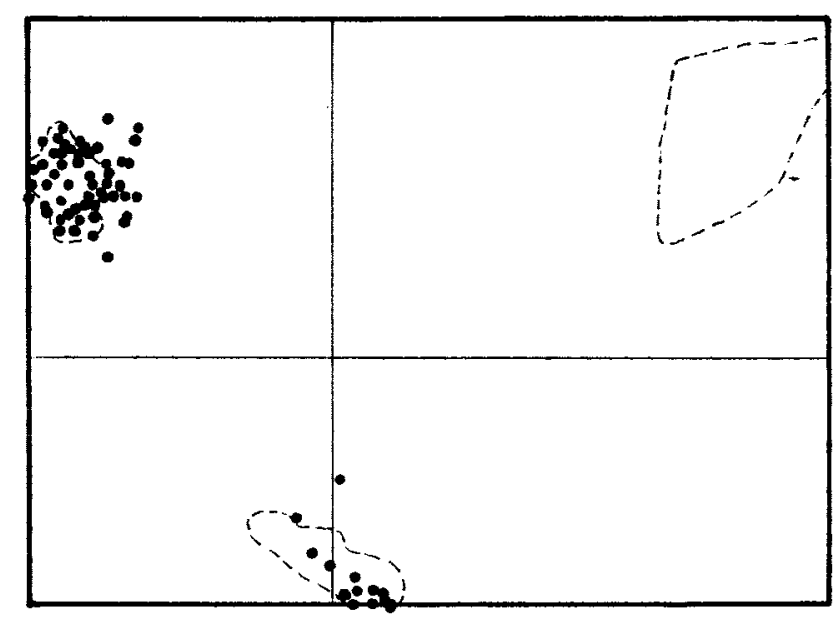

FIG. 13

Structure taxonomique des chênaies xéro-acidophiles.

\subsection{Les milieux hydromorphes (fig. 14)}

Le terme de sols hydromorphes désigne ici des sols à hydromorphie temporaire et à nappe perchée (pseudogley au sens large). Il s'agit de milieux humides et souvent acides, l'acidité provenant du matériau (sableux) ou de l'eau rendue réductrice et asphyxiante par la stagnation.

On y trouve essentiellement du chêne pédonculé, mais quelques sessiles peuvent apparaître à la faveur d'un micro-relief. 


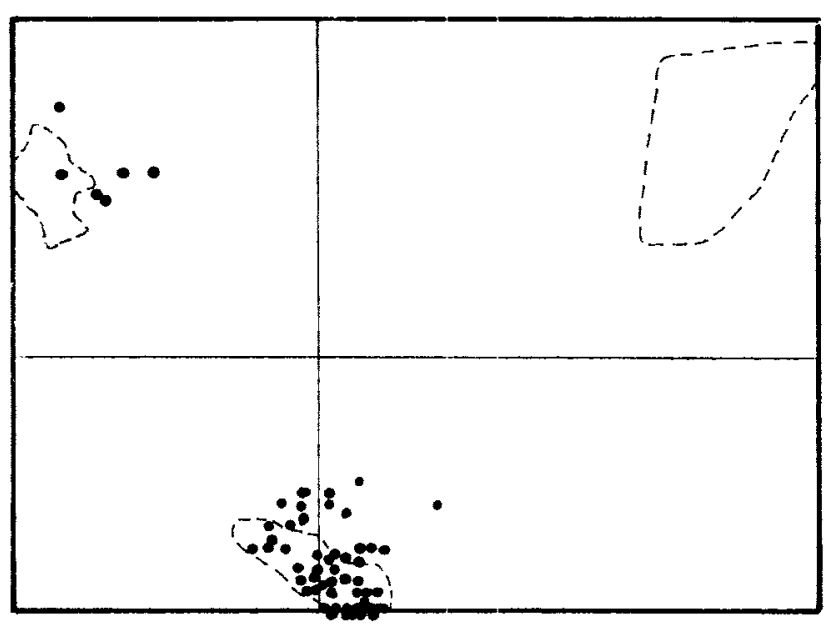

FIG. 14

Structure taxonomique des chênaies hydromorphes.

\subsection{Répartition du chêne pédonculé}

Le chêne pédonculé est présent dans tous les milieux, à l'état diffus ou en peuplements purs. Il se manifeste souvent comme un «parasite » dans la répartition autoécologique (fig. 9) et dans les images taxonomiques des populations. Cette ubiquité peut surprendre, mais il s'agit là d'un phénomène générale en France (sinon en Europe) que l'on interprête comme résultant de l'action anthropique. A faible densité, l'espèce apparaît à la fois conquérante et rustique, s'accommodant de tous les milieux :

- le chêne pédonculé a ainsi colonisé les espaces ouverts (forêts claires ou friches) ;

- il a aussi pu être introduit en plantations ou en compléments de régénération : les fructifications sont abondantes sur les arbres champêtres (panage), les glands sont gros et de belle apparence, se conservent dans l'eau, et le taux de germination est élevé, ce qui n'est pas le cas pour le sessile ; le semis exige très tôt la pleine lumière ; l'arbre est apprécié pour son fût droit et cylındrique;

- enfin, il a pu se maintenir sur ses acquis par suite de traitements sylvicoles extensifs : taillis simple pâturé pendant longtemps, puis taillis-sous-futaie depuis 150 ans au plus.

Le chêne pédonculé, par son caractère colonisateur, et à la faveur de l'action humaine, a donc débordé sa localisation "naturelle » au détriment du sessile.

Le chêne sessile paraît en outre désavantagé par une régénération moins performante, sinon moins abondante, dans certains milieux : glands intolérants à l'eau, avec une capacité germinative plus faible, ce qui pourrait expliquer sa rareté en stations hydromorphes (Shaw, 1968). De plus, ses glands petits et difficiles à conserver devaient être peu prisés par le forestier. 
Ces réflexions ont déjà pour l'essentiel été faites : BeCKER, LÉvy, 1983-1985 et plus anciennement (et tombées dans l'oubli): Turc, 1927 ; Demorlaine, 1927 ; Duhamel, 1984, aboutit aussi aux mêmes conclusions.

\subsection{Conclusion}

La confrontation de la taxonomie et de l'écologie a mis en évidence une double relation :

- celle d'une espèce avec ses préférences écologiques : autoécologie,

- celle d'une population avec son milieu : synécologie.

Le premier point a vérifié les exigences des trois espèces, et correspond à une nécessaire simplification de leur répartition, notamment pour la gestion forestière.

Le second est plus fondamental :

1) Il indique tout d'abord que la composition des peuplements a dû peu varier dans les siècles passés (taillis simples puis taillis-sous-futaie), contrairement à ce que l'on pourrait penser : une telle adéquation milieu/population ne peut être artificielle ni récente, sauf plantation.

Par contre, ces traitements clairs ont certainement favorisé le chêne pédonculé, qui apparait souvent comme un intrus dans les images taxonomiques des stations écologiques.

2) Les caractères morphologiques fins des populations de chênes indigènes obéissent de manière très précise aux indications des groupements végétaux. A la limite, la population de chênes se comporte comme une indicatrice stationnelle.

La différenciation morphologique des chênes correspond en fait à un continuum des chênaies qui obéissent elles-mêmes aux gradients écologiques. L'écologie vient ici apporter un éclairage indispensable à la taxonomie.

En particulier, les individus «intermédiaires» sessile $\times$ pédonculé et sessile $\times$ pubescent occupent des stations intermédiaires entre les exigences stationnelles des espèces-type. Cette corrélation, qu'ANDERSON, 1949 a appelé " hybridation par l'habitat », corrobore la thèse de l'introgression entre les trois chênes.

Il faut toutefois remarquer que le passage pédonculé-pubescent est très mal représenté sur la projection graphique taxonomique, ceci s'explique par le fait que les stations intermédiaires correspondantes sont peu fréquentes en Berry. La seule qui a pu être observée ne portait aucun chêne! Pour Guinier, 1970, c'est leur rareté qui explique celle des intermédiaires pédonculé-pubescent.

\section{Spéciation}

\subsection{Proximité et séparation génétiques}

Les chênes européens apparaissent aujourd'hui comme des espèces génétiquement proches mais distinctes. 
- Les essais de fécondation artificielle robur $\times$ petraea et robur $\times$ pubescens sont peu prometteurs et la viabilité des hybrides n'a pas été démontrée.

- L'identité chromosomique apparente du genre ne signifie nullement un appariement facile des chromosomes: un petit nombre de segments non homologues peut entraîner un taux élevé d'échecs de la fécondation croisée.

- On peut raisonnablement penser, au vu de la typologie des chênaies intermédiaires, et des expériences d'hybridation artificielle, que les croisements interspécifiques sont rares à l'heure actuelle dans la nature (sauf éventuellement les croisements petraea pubescens, mais les zones de transition où coexistent les deux espèces sont comparativement peu étendues).

Aucune pollution génétique interspécifique ne semble donc à craindre sous nos climats aujourd'hui en peuplements mélangés.

\subsection{Introgression}

Dans le cas de spéciation progressive, les espèces évoluent lentement, au sein d'une même aire. Les modifications sont d'abord physiologiques, puis morphologiques, et l'isolement sexuel n'intervient que dans la dernière phase.

Les facteurs écologiques sont les premiers et principaux dans l'isolement des populations. Lorsque localement ils disparaissent, des essaims d'hybrides se forment, avec introgression.

Cette évolution est lente et progressive, à l'échelle des temps géologiques. En ce qui concerne les chênes européens, quelques détails sont à nuancer : reux ;

- les individus intermédiaires sessile $\times$ pédonculé observés semblent peu vigou-

- leur rareté est sans doute en rapport avec un degré d'interfertilité variable entre les différentes formes. S'il y a effet dépressif de l'hybridation, il pourrait s'estomper graduellement à la faveur des croisements en retour avec l'un des parents. Tout semble indiquer à l'heure actuelle la supériorité de l'introgression sur l'hybridation (Cousens, 1965). L'existence d'hybrides interspécifiques viables et fertiles en climat ligérien ne peut être exclue, mais ces arbres seraient certainement en très faible nombre (SIGAUD, 1984).

Les grandes migrations (quaternaires notamment) ont probablement homogénéisé les espèces, alors que l'occupation en mosaïque du territoire, sur des surfaces parfois réduites, favoriserait la différenciation en formes, variétés, écotypes ou races locales.

Pour expliquer la plus grande fréquence d'intermédiaires en Europe du Nord (Scandinavie, Ecosse...), des auteurs (Cousens, 1965 ; Olsson, 1975) ont évoqué des facteurs climatiques, et le fait que le sessile y est en limite géographique, ce qui est supposé favoriser l'hybridation.

Rappelons enfin les travaux sur les chênes de l'Est des Etats-Unis, dont le polymorphisme a aussi été attribué à l'introgression (BENSON, 1962; JENSEN \& Esbaugh, 1976 ; Jensen, 1977 ; KNops \& Jensen, 1980). 


\section{Conclusion générale}

Ce travail s'insère dans un ensemble cohérent d'études et de réflexions sur les chênes européens.

1) Quercus robur, Quercus petraea et Quercus pubescens sont trois espèces à part entière. Les arbres réellement intermédiaires sont en faible nombre en Berry et sans doute dans une bonne partie de la France.

2) Ils ont des préférences écologiques bien distinctes, et la chênaie est le reflet du milieu naturel sauf plantation. Ainsi les arbres intermédiaires sont trouvés en peuplements mélangés dans des stations de transition.

Cela a été vérifié en taillis-sous-futaie, où la seule conséquence visible de l'action humaine est la présence ubiquiste du chêne pédonculé, colonisateur de milieux ouverts.

3) Le polymorphisme pourrait résulter de l'hybridation introgressive dans les milieux de transition, simultanée de la spéciation dans des stations plus extrêmes. Les populations introgressées représenteraient une adaptation aux conditions du milieu, expliquant la corrélation observée.

On peut conclure à l'heure actuelle à une hybridation limitée, et à une large introgression. La différenciation de races locales se superposerait à cette évolution.

En particulier:

- aucune pollution interspécifique n'est à craindre en peuplements mélangés, ce qui conditionne en grande partie la conduite actuelle à tenir dans certaines forêts ;

- la gestion forestière doit être attentive à la distinction des espèces de chênes, et à ses applications sylvicoles. Ce point fait à lui seul l'objet d'une publication à paraître dans la Revue Forestière Française.

Reçu le 21 janvier 1985.

Accepté en mars 1986.

\section{Summary}

The contribution of the Berry oaks to taxonomy and ecology

Microscopical observations of the morphology of the twigs, leaves and peduncles of 419 Oak trees were carried out in the sympatric and allopatric populations of Berry (Central France). Nineteen characters, divided into a total of 82 classes, were measured on each tree. Multivariate analysis of the data displayed 3 distinct poles connected by a few intermediates. These 3 poles correspond to Quercus robur L., Quercus petraea (Matt.) Liebl., and Quercus pubescens Willd. Multivariate analysis and the linear correlation of the characters allowed the discriminating taxonomic criteria to be distinguished.

The variation in peduncle and petiole lengths was studied at both the tree and population level. These analyses displayed discontinuities betwen Sessile and Pedunculate Oaks and allowed comparisons to be made with other studies made in France and elsewhere. study.

Similarly a phytoecological study allowed the study plots to be correlated with the taxonomic 
It was thus possible to provide evidence for the xerothermophilic nature of Downy Oaks and the hygrophilic nature of Pedunculate Oak. Sessile Oak appeared to be particularly tolerant of dry and acidic conditions.

The very rare intermediates at Berry were only present on neutral, mesophilic sites and completely absent on xero-acidophilic or hydromorphic sites. The Oak population distribution, then, appears to be related to ecological site types i.e. taxonomic speciation and ecological segregation are closely linked.

The distribution of Pedunculate Oak, present to at certain extent in a number of ecological sites, is likewise discussed.

Although interspectific gene exchange at Berry appears very limited today, the hypothesis of introgression appears, after all, to be plausible.

\section{Références bibliographiques}

ANDERSON E., 1949. Introgressive hybridization, J. Wiley, New York, $109 \mathrm{p}$.

Becker M., 1972. Quelques observations morphologiques chez les chênes sessile et pédonculé. Bull. Soc. Bot. Fr., 119 (3-4), 231-236.

Becker M., LÉvy G., 1983. Le dépérissement du chêne : Les causes écologiques. Exemple de la Forêt de Tronçais. R.F.F., 35 (5), 341-356.

Benson L., 1963. Plant Taxonomy: methods and principles. The Ronald Press Company, New York, $495 \mathrm{p}$.

Bidault M., 1971. Variation et spéciation chez les végétaux supérieurs. Doin, Paris, 117 p.

Camus A., 1938-1939. Monographie du genre Quercus. Tome Il (Sous-genre Euquercus). Paul Lechevallier, Paris.

Cousens J.E., 1965. The status of pedunculate and sessile oak in Britain. Watsonia, 6 (3), 161176.

Demorlaine J., 1927. La grande misère du chêne danis nos forêts françaises. R.E.F., 1927, 1-3.

Deret-Varcin E., 1983. Etude comparative de la qualité du bois de 3 types de chêne. Ann. Sci. For., 40 (4), 373-398.

Duhamel D., 1984. Statut écologique du chêne sessile et du chêne pédonculé dans le quart NordOuest de la France. Mémoire E.N.I.T.E.F. de $3^{e}$ année, 1983-1984.

Dupouey J.L., 1983. Analyse multivariable de quelques caractères morphologiques de chênes du Hurepoix. Ann. Sci. For., 40 (3), 265-282.

Gardiner A.S., 1970. Pedunculate and sessile oak : a review of the hybrid controvers. Forestry, 43 (2), 151-160.

Gathy P., 1969. Contribution à l'étude de la génétique des chênes. F.A.O., $2^{\mathbf{c}}$ conférence mondiale sur les arbres forestiers. Washington, Aug. 1969.

Guinier P., 1950. Qu'est-ce-que le chêne. Bull. Soc. For., Franche-Comté, 25 (2), 553-589.

JENSEN R.S., 1977. A preliminary numerical analysis of the red oak complex in Michigan and Wisconsin. Taxon, 26 (4), 399-407.

JenSen R.S., Essaugh N.H., 1976. Numerical taxonomic studies of hydridization in Quercus. Syst. Bot., 1, 1-19.

Jones E.W., 1959. Biological Flora of the British Isles: Quercus L., J. Ecol., 47, 169-222.

Jovanovic M. et al., 1973. Hybridations contrôlées intra et interspécifiques chez les chênes. Sumartsvo, 9-10, 3-14.

Kissuing P., 1977. Les poils des quatre espèces de chènes du Jura. Ber. Schweiz. Bot. Ges., 87 $(1 / 2): 1-18$.

Kissuing P., 1980a. Clef de détermination des chênes médioeuropéens. Ber. Schweiz. Bot. Ges., $90(1 / 2), 29-44$. 
Kissuing P., 1980b. Un réseau de corrélations entre les chênes du Jura. Ber. Schweiz. Bot. Ges, $90(1 / 2), 1-28$.

Kissling P., 1983. Les chênaies du Jura Central Suisse. Mémoires Institut Fédéral de Recherches Forestières, 59 (3), $387 \mathrm{p}$.

KNops J.F., JeNSEN J.J., 1980. Morphological and phenolic variation in a three species community of red oaks. Torrey Bot. club., 107, 418-428.

Kotschy T., 1864. Les chênes de l'Europe et de l'Orient. Rothschild, Paris.

Krahl-Urban J., 1959. Die Eichen. Hambourg et Berlin.

Lebreton O., 1976. Quelques données chimiotaxonomiques relatives aux Fagacées. Bull. Soc. Bot. Fr., 123, 293-298.

Moggi G., Paoli P., 1972. Ricerche sulle querce caducifoglie italiane. 1 - Sul valore di alcuni caratteri biometrici e morfologici. Webbia, 26 (2), 417-461.

Mourey J.M., 1979. Les chênes de la Vallée de l'Ognon. Mémoire E.N.I.T.E.F. de $3^{e}$ année, 1978-1979.

Olsson U., 1975. A morphological analysis of phenotypes in populations of Quercus (Fagaceae) in Sweden. Bot. Notiser, 128, 55-68.

Olsson U., 1976. Peroxydase isozymes in Quercus petraea and Quercus robur. Bot. Notiser, 128, 408-411.

Pellecuer B., 1976. Ecologie des chênes en forêt de Morimond. Thèse de doctorat de $3^{c}$ cycle. Université de Nancy I, juin 1976.

Rushton B.S., 1977. Artificial hybridization between Quercus robur L. and Quercus petraea Liebl. Watsonia, 11, 229-236.

Rushton B.S., 1978. Quercus robur and Quercus petraea : a multivariate approach to the hybrid problem. 1 - data acquisition, analysis and interpretation. Watsonia, 12, 81-101.

Rushton B.S., 1979. Quercus robur and Quercus petraea (...). 2 - The geographical distribution of population types. Watsonia, 12, 209-224.

Shutilov V.A., 1968. Hybridation introgressive et variabilité des chènes de Caucase. Bot. Zhurnal, 53 (2), 243-253.

Sigaud P., 1984. Les chênes du Berry. Mémoire E.N.I.T.E.F. de 3c année, 1983-1984.

Stefanoff B., Kostov K., 1962. Chênes et chênaies en Bulgarie: Caractères dendrologiques et écologiques. Acad. Sc. Agr., Bulgarie 1962, 25-29.

Turc ? ?, 1927. Note sur le dépérissement du chêne pédonculé dans le Nivernais. R.E.F. 1927 , 561-565.

Van Campo M., Elhal H., 1956. Etude comparative du pollen de quelques chênes. Bull. Soc. Bot. Fr., 103 (5-6), 254-290.

Wigston D.L., 1974. Cytology and genetics of oaks. "The British Oak". Bot. Soc. British Isles, $27-50$. 